\title{
Myopathy with abnormal structure and function of muscle mitochondria
}

\author{
W. C. HULSMANN, ${ }^{1}$ J. Bethlem, A. E. F. H. MEiJer, P. FleURy, \\ AND J. P. M. SCHELLENS
}

\begin{abstract}
From the Medical School, Rotterdam, the Departments of Neuropathology of the Neurological Clinic, Pathological Anatomy, the Neurological Clinic, and the Histological Laboratory, University of Amsterdam
\end{abstract}

When the rate of respiration of mitochrondria in the presence of inorganic phosphate cannot be increased by adding adenosine diphosphate (A.D.P.) but these particles can still couple respiration to phosphorylation of A.D.P. to adenosine triphosphate (A.T.P.), the state of respiration is called 'loosely coupled'.

The first patient in whom such a loosely coupled state of oxidative phosphorylation of the muscle mitochrondria was found was described by Ernster, Ikkos, and Luft (1959). The patient suffered from generalized myopathy with progressive asthenia and severe hypermetabolism. The structure of the muscle mitochondria was markedly altered as shown by electron microscopy (Luft, Ikkos, Palmieri, Ernster, and Afzelius, 1962).

The second patient with the same biochemical disturbance of oxidative phosphorylation of the muscle mitochondria was described by Van Wijngaarden, Bethlem, Meijer, Hülsmann, and Feltkamp (1967). This patient showed a proximal myopathy without hypermetabolism. Histological examination of a muscle biopsy revealed subsarcolemmal zones that contained small granules. In these zones, an increased activity of oxidative enzymes was demonstrated. Electronmicroscopic examination showed the presence of pathological mitochondria. Large spherical, rod-shaped or annular mitochondria were observed. The morphology of the pathological mitochondria differed in many respects from that observed by Luft et al. (1962), who, in addition, found peculiar inclusions in the mitochondria. Giant mitochondria with rectangular and other inclusions were found in the muscle of a child with proximal weakness by Shy, Gonatas, and Perez (1966), in a disorder which they termed megaconial myopathy. In this case, data on biochemical studies of oxidative phosphorylation of the morphologically altered muscle mitochondria were not given.

'Temporary address: Dijkzigt Hospital, Rotterdam, the Netherlands.
A third case in which morphologically altered muscle mitochondria and a loosely coupled state of oxidative phosphorylation were present will now be described.

\section{CLINICAL INVESTIGATIONS}

The patient's parents were both 27 years old and in good health. The mother was white; the father was a Negroid from South Africa. An elder sister was in good health. No myopathies existed in the parental families and there was no parental consanguinity. Pregnancy and parturition had taken a normal course.

The patient started to walk when 1 year old and no abnormal signs or symptoms were noted until the age of 2 , when her gait became uncertain. A few months later she had to be carried a good deal, and she could no longer rise from a sitting position without assistance. She was 27 months old when admitted to hospital.

At examination we saw a Negroid girl who gave no impression of illness. She displayed a staggering gait and could not rise unassisted from a sitting or recumbent position. The muscles of the shoulder girdle, pelvic girdle, upper arms, and thighs were paretic, atrophic, and hypotonic. There was no hypertrophy of the calf muscles or any other muscles. Examination of the internal organs disclosed no abnormality, and radiographs of the skull, spine, chest, and pelvis were normal. Both the E.E.G. and the E.C.G. were without abnormalities. The E.M.G. (Dr W. J. M. Hootsmans) was derived bilaterally from the gluteus medius and quadriceps femoris muscles with the aid of concentric needle electrodes. The patient's resistance limited the scope of this examination, which disclosed no unequivocal abnormalities.

LABORATORY FINDINGS The Hb concentration was $12 \cdot 3$ g. $/ 100 \mathrm{ml}$. White cell count and E.S.R. were normal. Haematocrit value was $39 \%$. Blood urea was $230 \mathrm{mg} . / 1$., sodium $134 \mathrm{mEq} / \mathrm{l}$, potassium 4.3 mEq./l., calcium $10.2 \mathrm{mEq} . / \mathrm{l}$., cholesterol $348 \mathrm{mg} . / 100 \mathrm{ml}$; ; serum lactic acid concentration $32 \mathrm{mg}$. $/ 100 \mathrm{ml}$.

Serum creatine phosphokinase and aldolase activities amounted to $5.0 \mathrm{mU} / \mathrm{ml}$. and $4.8 \mathrm{mU} / \mathrm{ml}$., respectively. S.G.O.T. was 33U and S.G.P.T. 10U. 
Urinary creatinine excretion was $190-230 \mathrm{ml} . / 1$. and creatine excretion 300-500 mg./l.

It was impossible to collect 24 -hour urines. The $\alpha$ amino-nitrogen excretion was $22 \mathrm{mg}$. $/ 100 \mathrm{ml}$. The cerebrospinal fluid was normal; serological reactions in serum and cerebrospinal fluid were negative. The B.M.R., as estimated on the basis of oxygen consumption (Dr J. Muller), was $-19 \%$.

\section{MORPHOLOGICAL STUDIES}

MATERIAL AND METHODS For light microscopy specimens from the right and the left vastus lateralis were fixed in Heidenhain's fixation fluid and then embedded in paraffin for cutting longitudinal and transverse sections which were stained with haematoxylin-eosin and Gomori's trichrome stain.

For histochemical investigation other parts of the specimens were rapidly frozen by immersing small blocks of tissue in isopentane cooled to $-150^{\circ} \mathrm{C}$. with liquid nitrogen. Longitudinal and transverse serial sections were cut in a cryostat. Fixed or unfixed sections were stained for the activities of the following oxidative enzymes: N.A.D.H.-tetrazolium reductase, N.A.D.P.H.tetrazolium reductase, lactate dehydrogenase, malate dehydrogenase (N.A.D. ${ }^{+}$), malate dehydrogenase (N.A.D.P. ${ }^{+}$) decarboxylating, isocitrate dehydrogenase (N.A.D. ${ }^{+}$), isocitrate dehydrogenase (N.A.D.P. ${ }^{+}$), glyceraldehyde-3-phosphate dehydrogenase, glycerol-3-phosphate dehydrogenase (N.A.D. ${ }^{+}$, glycerol-3-phosphate oxidase (menadione), glucose-6-phosphate dehydrogenase, glutamate dehydrogenase, 3-hydroxybutyrate dehydrogenase, succinate dehydrogenase, and monoamine oxidase. Other enzyme activities tested were: alpha-glucan phosphorylase $a$ and $b$, adenosine triphosphatase (Mg-activated), myosin-adenosine triphosphatase, 5'-nucleotidase, fructose-1,6-diphosphate aldolase, peroxidase, cytochrome $c$ oxidase and acid phosphatase. Glycogen was estimated by the periodic-acidSchiff reaction before and after digestion by diastase. The histochemical methods used are described in a previous paper. (Van Wijngaarden et al., 1967).

For electron microscopy, biopsy specimens in the order of $1 \mathrm{c} . \mathrm{mm}$ were fixed for three hours at $0-4^{\circ} \mathrm{C}$. in $6 \%$ glutardialdehyde dissolved in $0.1 \mathrm{M}$ cacodylate buffer $p H 7.2$ with $0.22 \mathrm{M}$ sucrose added. The fragments were then kept for a few hours in the same fluid without glutardialdehyde and were postfixed in buffered aqueous $\mathrm{OsO}_{4}$ according to Millonig(1961): $1 \% \mathrm{OsO}_{4}, 0 \cdot 1 \mathrm{M} \mathrm{NaH}_{2} \mathrm{P}$ $\mathrm{O}_{4}, 0 \cdot 1 \mathrm{M} \mathrm{NaOH}, 0.03 \mathrm{Mglucose}$. After dehydration in an alcohol series, the tissue was embedded in Epon. Sections were cut on an LKB ultramicrotome and, after 'staining' with lead tartrate, studied in a Siemens Elmiskop Ia with a $400 \mu$ condensor aperture and a $50 \mu$ objective aperture.

RESUlTs Histopathological studies disclosed two types of muscle fibre: those with a normal diameter and structure, and those with a very small diameter and frequently of more polygonal shape in transverse section (Fig. 1). These fibres were found among the normal fibres in no recognizable pattern and

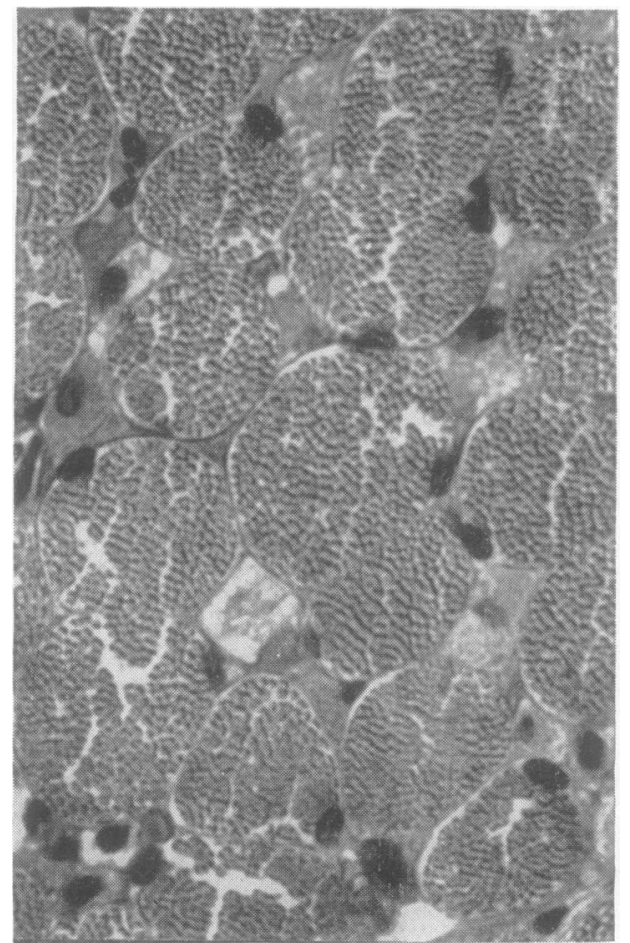

FIG. 1. Transverse section showing fibres with normal diameter and fibres with a very small diameter. Gomori's trichrome $\times 250$.

amounted to about $40 \%$ of the total number of fibres. The structure of the fibres of small diameter showed pronounced changes, sometimes throughout their entire length (Fig. 2) and in others only through part of their length. Transverse sections showed that the entire cut surface of the fibres was affected; occasional fibres showed a preponderance of subsarcolemmal changes. The affected fibres contained very finely granulated material, in which sometimes only a few myofibrils were observed. These areas showed marked proliferation of vesicular nuclei with prominent nucleoli. The affected muscle fibres also showed a conspicuous number of capillaries. There were no signs of phagocytosis. Endomysial fat tissue and connective tissue were slightly increased. Blood vessels and intramuscular nerves showed no abnormalities. Histochemical examination revealed that the histologically normal muscle fibres showed medium enzyme activity with all techniques used. The fibres of small diameter showed a very high activity of the oxidative enzymes (Fig. 3). The aldolase activity was likewise high. The activity of $\alpha$-glucan phosphorylase a and $b$ was low in some and high in other fibres. The periodic-acid- 


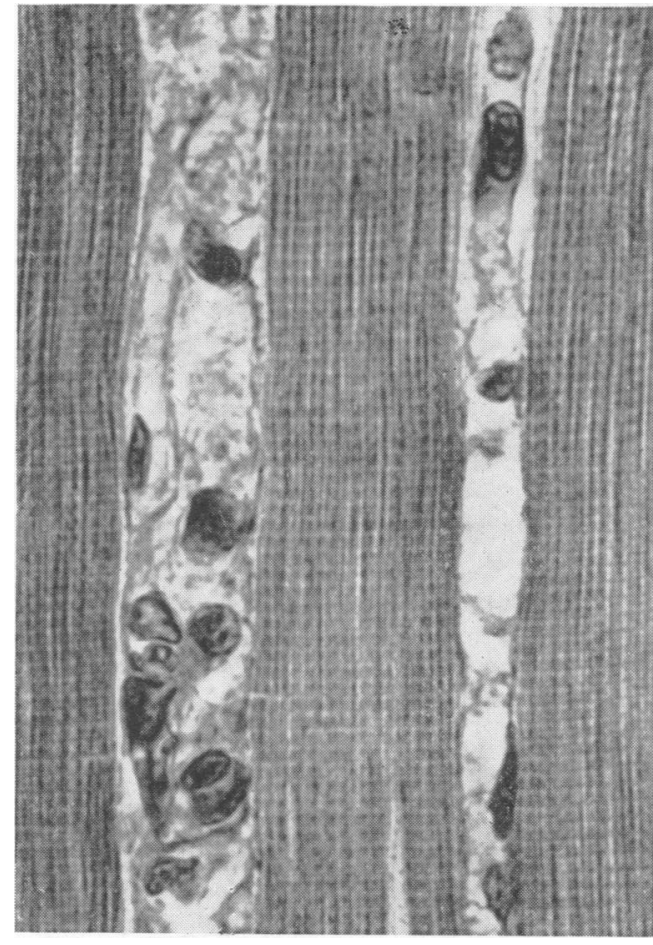

FIG 2

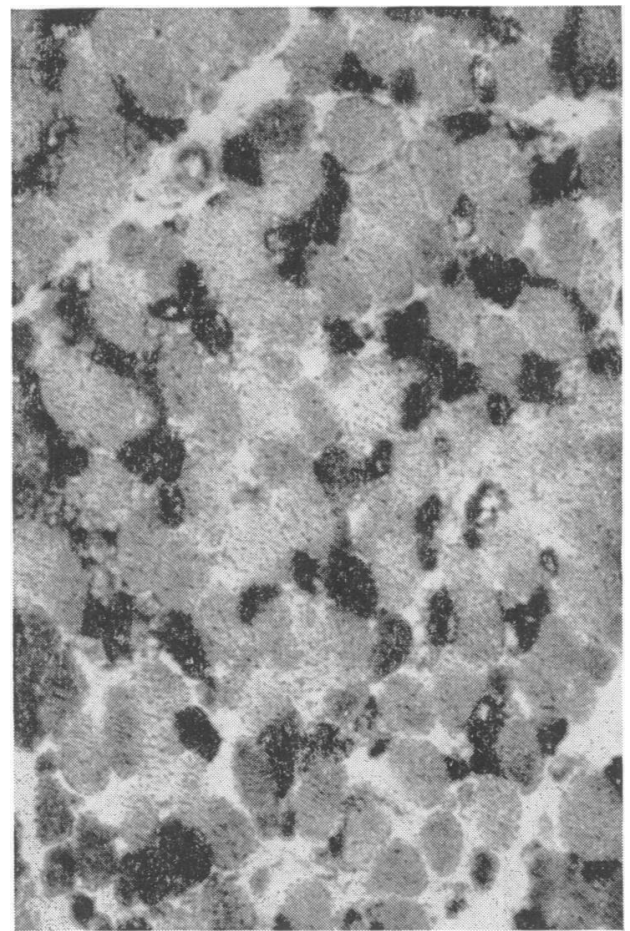

FIG. 3



FIG. 4

FIG. 2. Longitudinal section showing two small fibres in which very finely granulated material and vesicular nuclei are seen. Gomori's trichrome $\times 250$.

FIG. 3. Transverse section. Medium activity of the fibres with normal diameter and a very high enzyme activity of the small fibres. N.A.D.H.-tetrazolium reductase $\times 100$.

FIG. 4. Adjacent to a normal fibre (right) a fibre containing numerous mitochondria is seen. No myofibrils are present $\times 12,000$. 




FIG. 5. (a) Abnormal mitochondria with irregular inner structure $\times 22,000$.

(b) Giant mitochondrion with vacuoles, concentric lamellae, and dense membrane configurations $\times 12,500$.

(c) Part of a formation of dense coarse lamellae $\times 8,500$.

(b)

Schiff reaction was negative in some and positive in other pathological fibres. There was medium activity of $\mathrm{Mg}$-activated adenosine triphosphatase, but no or only very slight activity of calciumactivated adenosine triphosphatase catalysed by the myofibrils.

The unusual light microscopic aspect of some muscle fibres was confirmed at electron microscopy. These fibres contained very few, if any, myofilaments; instead they contained numerous mitochondria, which in many respects differed from normal ones
(Fig. 4), and were situated in the sarcoplasm without any apparent orientation. In some areas they did not seem to differ very greatly from the mitochondria in the more normal fibres, although their internal structure was always tubulo-vesicular, whereas in the apparently normal fibres many mitochondria with classical cristae were present. Besides these more or less normal ones, many obviously abnormal mitochondria were seen which were much larger than normal (giant mitochondria) and of quite irregular shape (Figs. 5a, 5b, and 6d). The most striking 
(a)

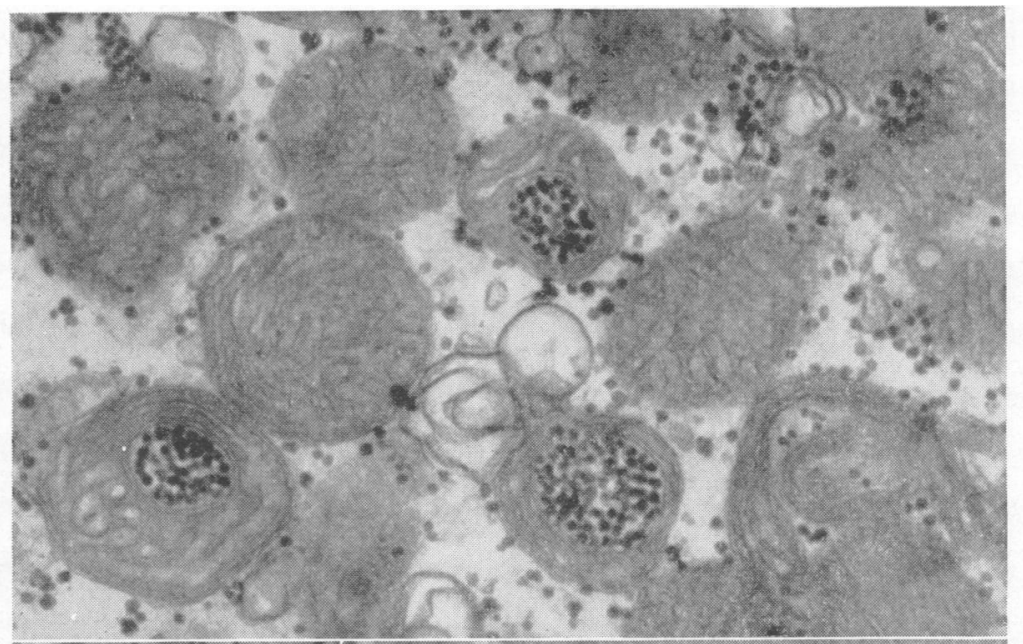

FIG. 6. (a) Mitochondria which seem to contain glycogen particles enclosed by a double membrane $\times 48,000$.

(b) Orderly packed structure of unknown nature in the sarcoplasm $\times 45,000$.

(c) Part of a mitochondrion with concentric lamellar cristae (top right) and a mitochondrion-like structure containing glycogen $\times$ 33,000 .

(d) Giant mitochondrion of very irregular shape, with vacuoles and concentric lamellae $\times 18,000$.

(b)

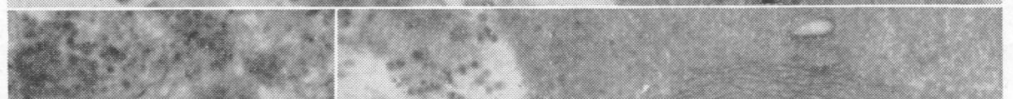

(d)



aspect of these abnormal mitochondria was their internal organization. In most of them there were, apart from a tubulo-vesicular membrane system, one or more of the following unusual components: vacuoles (Figs. 5b and 6d), concentric lamellae, resembling cristae (Figs. 5a, 5b, 6c, and 6d), membrane configurations of high electron density (Figs. 5a and 5b). In a few mitochondria there were particles which, as judged by their dimensions (250$300 \AA$ ) and their electron density after 'lead-staining', may be considered to be $\beta$-glycogen particles
(Figs. 6a and 6c). The particles were enclosed by what seemed to be a normal mitochondrial double membrane; the most plausible explanation of their presence within the mitochondria seems to be that they were 'caught' when mitochondria developed irregular shapes. In between the mitochondria bulky accumulations of very electron dense, coarse lamellae were sometimes seen (Fig. 6c). In one fibre a curious honeycomb-like structure was observed (Fig. 6b), the nature of which was not clear. 
BIOCHEMICAL STUDIES

MATERIAL AND METHODS A specimen $(2 \mathrm{~g}$.) of the left vastus lateralis muscle was finely minced and homogenized in ice-cold isolation medium. The mitochondria were isolated by differential centrifugation of the homogenate, using in principle the method of Luft et al. (1962). The rates of oxidation of pyruvate and glutamate by the isolated mitochondria were measured by the Warburg technique, employing differential manometers, provided with small flasks with centre wells and sidearms. The main compartments of the flasks contained the following components: $25 \mathrm{mM}$ glucose, $50 \mathrm{mM} \mathrm{KC1,} 25 \mathrm{mM}$ Tris$\mathrm{HCl}$ buffer of $p \mathrm{H} \mathrm{7.6,25} \mathrm{mM}$ potassium-phosphate buffer of $p \mathrm{H} \mathrm{7.4,} \mathrm{0.5} \mathrm{mM} \mathrm{E.D.T.A.,} \mathrm{0.25} \mathrm{mM} \mathrm{cytochrome}$ c, $5 \mathrm{mM} \mathrm{MgC1}_{2}, 0.5 \mathrm{mM}$ A.T.P. and oxidizable substrates. The centre wells contained $\mathrm{KOH}$ and a paper wick to bind $\mathrm{CO}_{2}$, and the side arms contained $500 \mathrm{mU}$ of hexokinase. The reactions were started by the addition of $0.5 \mathrm{mg}$. of mitochondrial protein to the main compartments. The flasks were then attached to differential manometers, shaken at $25^{\circ} \mathrm{C}$., and readings taken at regular intervals. After 25 minutes the hexokinase was added from the side arms, so that A.D.P. could now be generated continuously from A.T.P. Thirty-five minutes later the reactions were stopped by the addition of perchloric acid. Glucose-6-phosphate was determined in the deproteinized, neutralized extracts with glucose-6phosphate dehydrogenase and N.A.D.P. ${ }^{+}$.

Phosphorylation accompanying oxidation of pyruvate and glutamate was also measured. The techniques used and the results obtained in six control patients are described in detail in the paper by Van Wijngaarden et al. (1967).

Finally the isoenzyme pattern of lactic acid dehydrogenase was determined in a muscle specimen by agar electrophoresis.

RESULTS The rates of oxygen uptake in the presence and absence of phosphate acceptor and the rate of phosphorylation with pyruvate or glutamate as oxidizable substrates are shown in Table I. It can be

\section{TABLE I}

ABSENCE OF STIMULATION OF RESPIRATION BY THE ADDITION OF PHOSPHATE ACCEPTOR

\begin{tabular}{|c|c|c|c|}
\hline \multirow[t]{3}{*}{ Respiratory Substrate(s) } & \multicolumn{3}{|c|}{ Phosphate Acceptor } \\
\hline & \multirow{2}{*}{$\frac{\text { Absent }}{\Delta \mathrm{O}}$} & \multicolumn{2}{|c|}{ Present } \\
\hline & & $\Delta \mathbf{O}$ & $\mathbf{\Delta P}$ \\
\hline $\begin{array}{l}20 \mathrm{mM} \text { Pyruvate }(+0.3 \mathrm{mM} \text { L-malate }) \\
20 \mathrm{mM} \text { Glutamate }(+0.3 \mathrm{mM} \text { L-malate })\end{array}$ & $\begin{array}{l}4 \cdot 0 \\
4 \cdot 3\end{array}$ & $\begin{array}{l}4 \cdot 0 \\
4 \cdot 5\end{array}$ & $\begin{array}{l}7 \cdot 6 \\
8 \cdot 6\end{array}$ \\
\hline
\end{tabular}

The $\Delta O$ (in $\mu A t / m g$. protein/h) was corrected for the (almost negligible amount of) oxygen taken up in the absence of added pyruvate or glutamate but in the presence of malate.

The $\Delta \mathbf{P}$ (in $\mu \mathrm{mol}$ glucose-6-phosphate $/ \mathrm{mg}$. protein $/ \mathrm{h}$ ) was corrected for the amount of G-6-P formed in the presence of malate only as the substrate. seen that the addition of phosphate acceptor does not alter the rate of oxidation although phosphorylation occurs. This phenomenon indicates that oxidative phosphorylation catalysed by the mitochondria is loosely coupled. The L.D.H. isoenzyme pattern showed a significant diminution of L.D.H.-5.

\section{COMMENT}

At the age of 2, the patient developed a rapidly progressive proximal myopathy involving the humeroscapular and pelvic muscles. Histological examination disclosed numerous muscle fibres of small diameter, which contained fine granules. This granular material showed a very high activity of oxidative enzymes.

Histochemical examination disclosed no myosin A.T.P.ase in these small fibres. Electron microscopy revealed the presence of large and abnormal mitochondria in these fibres. Mitochondria isolated from a homogenate of a biopsy from a vastus lateralis muscle showed a loosely coupled state of oxidative phosphorylation. We considered the possibility that this biochemical finding might have resulted from errors made in the isolation of the mitochondria and/or the determination of oxidative phosphorylation. However, eight control biopsies (six of which have been described by Van Wijngaarden et al.) were examined in exactly the same manner as the present case and showed, with glutamate and pyruvate as respiratory substrates, an increase of oxygen consumption upon the addition of phosphate acceptor which varied from $2 \cdot 3$ to fivefold.

The patient of Luft et al. showed severe hypermetabolism (B.M.R. ranging from $+140 \%$ to + $210 \%$ without hyperthyroidism, and is to be expected when large numbers of mitochondria show a loosely coupled state of oxidative phosphorylation. The case of Van Wijngaarden et al. and the present case showed the same biochemical disorder, although no hypermetabolism was found. This may be explained by a relatively small overall number of muscle mitochondria being in a loosely coupled state. It may well be that the changes in structure and function of the muscle mitochondria in the present case are the result of a biochemical disorder outside the mitochondria. Swelling of mitochondria was seen in electron microscopic studies of muscle biopsies from 34 patients with muscular dystrophy of various clinical types, and from five other patients with neurogenic atrophy by Fisher, Cohn, and Danowski (1966). These authors also suggest that it is most unlikely that these ultrastructural alterations represent a significant causative factor in the pathogenesis of the dystrophic disorders.

A tubulo-vesicular membrane system and an 
arrangement of the cristae in the form of concentric lamellae are described for normal mitochondria, e.g. by Zelander (1959) in mouse adrenal cortex. As to the giant mitochondria observed in the present case, it must be pointed out that mitochondrial gigantism is observed in various conditions and seems to be an aspecific response to different abnormal circumstances (Rouiller and Jézéquel, 1963; Svoboda, Grady, and Higginson, 1966; Volk and Scarpelli, 1966). With respect to the internal concentric lamellae and dense membrane configurations, it is of interest that some authors describe similar formations in degenerative mitochondria (Bovis, Kasten, and Okigaki, 1966; Jézéquel, 1959; Kisch, Cavusoglu, and Marangoni, 1959; de Robertis and Sabatini, 1958; Volk and Scarpelli, 1966).

It seems possible that the accumulations of dense coarse lamellae lying free in the sarcoplasm are remnants of degenerated mitochondria, since myelin-like figures are often associated with cellular regression and the membrane configurations observed inside the mitochondria may be interpreted as indications of degeneration.

It should be stressed that the irregular myelin-like figures may be fixation artifacts. According to Oláh and Röhlich (1966), glutaraldehyde fixation can cause this type of formation.

The pictures of glycogen in mitochondria agree with those obtained by Fain-Maurel (1966) from a mollusc gland; both in the material described by this author and in the present case, glycogen appears to be enclosed by the double mitochondrial membrane (Fig. 6a).

The present report is the second from our group to describe a loose coupling of the isolated skeletal muscle mitochondria. The fact that these two cases were seen within a period of six months may mean that this kind of phenomenon is not extremely rare. It seems probable that more of these cases will be found when morphological studies are correlated with biochemical investigations, even in the absence of hypermetabolism.

\section{SUMMARY}

The case is described of a 27 -month-old girl suf- fering from a myopathy involving the proximal muscles. Histopathological studies revealed the presence of muscle fibres of very small diameter, which contained finely granulated material. A very high activity of the oxidative enzymes was seen in these small fibres. Electron microscopy showed numerous mitochondria, many of which were of abnormal size and shape. Biochemical studies showed that the isolated mitochondria were loosely coupled.

\section{REFERENCES}

Bovis, R., Kasten, F. H., and Okigaki, T. (1966). Electron microscopic study of the toxic effect of sodium fluoroacetate on rat myocardial cultures. Exp. Cell Res., 43, 611-621.

Ernster, L., Ikkos, D., and Luft, R. (1959). Enzymic activities of human skeletal muscle mitochondria: a tool in clinical metabolic research. Nature (Lond.), 184, 1851-1854.

Fain-Maurel, M. A. (1966). Localisations intramitochondriale et intracisternale de glycogène monoparticulaire. C. R. Acad. Sci. Paris série D., 263, $1107-1110$.

Fisher, E. R., Cohn, R. E., and Danowski, T.S. (1966). Ultrastructural observations of skeletal muscle in myopathy and neuropathy with special reference to muscular dystrophy. Lab. Invest., $15,778-793$.

Jézéquel, A. M. (1959). Dégénérescence myélinique des mitochondries de foie humain dans un épithélioma du cholédoque et un ictère viral. J. Ultrastruct. Res., 3, 210-215.

Kisch, B., Cavusoglu, M., and Marangoni, B. A. (1959). Electron microscopic changes in human heart in cardiac failure. Exp. Med. Surg., 17, 85-100.

Luft, R., Ikkos, D., Palmieri, G., Ernster, L., and Afzelius, B. (1962). A case of severe hypermetabolism of nonthyroid origin with a defect in the maintenance of mitochondrial respiratory control: a correlated clinical, biochemical and morphological study. J. clin. Invest., 41, 1776-1804.

Millonig, G. (1961). A modified procedure for lead staining of thin sections. J. biophys. biochem. Cytol., 11, 736-739.

Oláh, I., and Röhlich, P. (1966). Peculiar membrane configurations after fixation in glutaraldehyde. Acta biol. Acad. Sci. Hung., 17, 65-74.

De Robertis, E., and Sabatini, D. (1958). Mitochondrial changes in the adrenocortex of normal hamsters. J. biophys. biochem. Cytol., 4, 667-678.

Rouiller, Ch., and Jézéquel, A. M. (1963). Electron microscopy of the liver. In The Liver vol. I, edited by $\mathrm{Ch}$. Rouiller, pp. 195-264. Academic Press, New York.

Shy, G. M., Gonatas, N. K., and Perez, M. (1966). Two childhood myopathies with abnormal mitochondria. I. Megaconial myopathy. II. Pleoconial myopathy. Brain, 89, 133-158.

Svoboda, D., Grady, H., and Higginson, J. (1966). The effects of chronic protein deficiency in rats. II. Biochemical and ultrastructural changes. Lab. Invest., 15, 731-749.

Volk, T. L., and Scarpelli, D. G. (1966). Mit schondrial g:gantism in the adrenal cortex following hypophysectomy. Ibid., 15, 707-715.

Van Wijngaarden, G. K., Bethlem, J., Meijer, A. E. F. H., Hülsmann, W. Ch., and Feltkamp, C. A. (1967). Skeletal muscle diseese with abnormal mitochondria. Brain, 90, 577-592.

Zelander, T. (1959). Ultrastructure of the mouse adrenal cortex. An electron microscopical study in intact and hydrocortisonetreated male adults. J. Ultrastruct. Res., suppl. 2. 